\title{
Fibra de coco e adubação foliar no crescimento e na nutrição de Cryptanthus sinuosus
}

\author{
Janie M Jasmim ${ }^{1}$; Regiane R V Toledo ${ }^{1}$; Leonardo A Carneiro ${ }^{2}$; Elisabeth Mansur ${ }^{2}$ \\ ${ }^{1}$ UENF-CCTA-LFIT, Av. Alberto Lamego, 2000, 28013-602 Campos dos Goytacazes-RJ; ${ }^{2}$ UERJ-IB-Dep ${ }^{\text {to }}$ Biologia Celular e Genética; \\ E-mail: janie@uenf.br
}

\begin{abstract}
RESUMO
Plantas de Cryptanthus sinuosus foram cultivadas em três substratos (mesocarpo de coco - MC; mesocarpo de coco lavado MCL e xaxim-X), visando comparar o efeito da fibra de coco verde, como substrato, àquele da fibra de xaxim sobre o crescimento e teores de nutrientes das plantas, sob três níveis de adubação foliar: 50, 100 e $150 \%$ de solução contendo $210 \mathrm{mg} \mathrm{L}^{-1}$ de N, $31 \mathrm{mg} \mathrm{L}^{-1}$ de P e $234 \mathrm{mg} \mathrm{L}^{-1}$ de K. O experimento constou de um fatorial em delineamento de blocos ao acaso, com quatro repetições e três plantas por parcela, uma planta por vaso e conduzido em casa de vegetação. O número de folhas, diâmetro da roseta, massa da matéria seca (MS) de folhas e raízes e teores de nutrientes foram avaliados. As plantas cultivadas em X apresentaram valores de MS de folhas e de raízes mais altos que aquelas em MC, mas não diferiram estatisticamente daqueles observados nas plantas em MCL. As plantas cultivadas em MC e MCL apresentaram características semelhantes indicando que a alta condutividade elétrica encontrada no substrato $\mathrm{MC}$ não prejudicou o acúmulo de matéria seca. As plantas cultivadas em MC e MCL apresentaram menores teores foliares de N, P, S que as cultivadas em X, porém com coloração e arquitetura foliares mais atrativas. Os diferentes níveis de adubação utilizados não tiveram efeito diferenciado sobre as características de crescimento, nem sobre os teores de nutrientes nas plantas.
\end{abstract}

Palavras- chave: bromélia, casca de coco, substrato, xaxim.

\begin{abstract}
Coconut fiber and foliar fertilization on the growth and nutrition of Cryptanthus sinuosus

Cryptanthus sinuosus plants were grown in three substrates (coconut mesocarp - MC, rinsed coconut mesocarp - MCL and tree fern fiber-X) aiming to compare the effect of green coconut fiber as substrate to tree fern fiber on the plant growth and nutrient contents, under three foliar fertilization levels $(50,100$ and $150 \%$ of a solution containing $210 \mathrm{mg} \mathrm{L}^{-1}$ of $\mathrm{N}, 31 \mathrm{mg} \mathrm{L}^{-1}$ of $\mathrm{P}$ and $234 \mathrm{mg} \mathrm{L}^{-1}$ of $\mathrm{K}$ ). The experimental design was a factorial scheme of randomized blocks with four replications and three plants per plot, one plant per pot, and was carried out in a greenhouse. The number of leaves, rosette diameter, leaf and root dry matter mass and nutrient contents were evaluated. Plants grown in $\mathrm{X}$ presented higher leaf and root dry matter mass values than those grown in MC, but did not differ from those observed on plants in MCL. The plants grown in MC and MCL showed similar characteristics indicating that the high electric conductivity of MC did not impair dry matter accumulation. The plants grown in MC and MCL showed lower N, P, S leaf contents than those grown in $\mathrm{X}$, nevertheless they showed more attractive leaf color and architecture. The levels of fertilization did not have differential effects either on the growth characteristics or on the plant nutrient contents.
\end{abstract}

Keywords: bromeliad, coconut husk, substrate, tree fern.

(Recebido para publicação em 1 de abril de 2005; aceito em 30 de agosto de 2006)

\begin{abstract}
$\mathrm{A}_{\mathrm{s}}$ floricultura abrange o cultivo e a exploração de plantas ornamentais, desde flores de corte ou secas, plantas em vaso, folhagens frescas ou desidratadas, até a produção de arbustos e árvores de grande porte. No Rio de Janeiro, as bromélias destacam-se como plantas ornamentais de grande potencial comercial. As plantas da família Bromeliaceae destacam-se por suas belas cores e formas, inflorescências, disposição das folhas, ocorrência em diversos habitats, e importância ecológica como abrigo, fonte de água e alimento para diversos animais.

Nos últimos anos tem crescido a importância econômica das bromélias, com sua utilização em projetos paisagísticos, devido à sua grande beleza, resistência e praticidade no manuseio, sendo usadas na decoração de jar-
\end{abstract}

dins e de interiores e em floriculturas por todo o Brasil. A comercialização é expressiva, para colecionadores e comerciantes, o que incentiva a coleta ilegal de espécies de maior beleza, acarretando a redução de populações naturais e aumento do número de espécies em risco de extinção (Melo, 1996). Segundo informações de representante do Instituto Estadual de Florestas (IEF), no ano de 2000 foram apreendidas mais de 3000 bromélias "clandestinas" nos morros na região de Guaratiba (Planta-Rio, 2001). Desta forma, o incentivo à profissionalização do cultivo dessas espécies e de outras plantas, constitui uma medida importante para a redução do extrativismo.

O gênero Cryptanthus é tipicamente nativo, sendo encontrado sempre em habitats terrestres ou rupícolas ao lon- go da costa na região de restinga da Mata Atlântica, bem como na caatinga e restinga (Cândido, 1995). Cryptanthus sinuosus foi descrita em 1955, baseada na descrição original de $C$. undulatus Otto e Dietrich, nomen illegitimum, como uma espécie terrestre e endêmica do Estado do Rio de Janeiro, encontrada em afloramentos rochosos no mar, bem como na floresta de restinga (Cândido, 1995). Suas características de porte reduzido, exigência de pouca luz e produção de flores delicadas, conferem à espécie um grande potencial ornamental para interiores.

Uma característica importante para a vida das bromélias é a presença de escamas ou tricomas cobrindo toda sua superfície foliar, ou formando bandas ou listras foliares, que podem absorver, por capilaridade, a umidade presente na at- 
mosfera levando-a ao parênquima (Rauh, 1990). Os tricomas, em geral, têm forma de escama peltada e consistem de um talo e escudo. O talo é uma coluna diminuta de células que liga os tecidos da epiderme ao escudo carregando água e nutrientes minerais do meio externo para o interno. O escudo é paralelo à superfície da folha e consiste de um disco central de células vivas com um anel externo de células mortas, o qual absorve nutrientes no meio ambiente. $\mathrm{O}$ disco atua como um plug fechando a concavidade no qual o talo está inserido e protegendo-o contra o ressecamento. A capacidade de absorção para diferentes tipos de tricomas depende da evolução da planta, morfologia dos tricomas e sua densidade nas folhas (Leme \& Marigo,1993). Na sub-família Bromelioideae, à qual pertence o gênero Cryptanthus, um pequeno disco composto por quatro células ocupa o centro do escudo que, com freqüência neste gênero, tem forma circular e margens regulares; tricomas presentes na base foliar, pelo menos em alguns casos, absorvem nutrientes acumulados em áreas adjacentes (Benzing, 2000).

Para o cultivo de plantas ornamentais, preconiza-se a utilização de recipientes (sacos plásticos, bandejas de isopor, vasos plásticos, etc.) e substratos específicos.

O xaxim, extraído da planta Dicksonia sellowiana, permaneceu como base para o preparo de substratos de várias espécies de plantas ornamentais no Brasil, inclusive bromélias, por muito tempo. Devido ao extrativismo desenfreado, as plantas de xaxim encontram-se em processo de extinção e, atualmente, têm sua extração e comercialização de seus derivados proibidos (Brasil, 2001), acarretando uma forte demanda por materiais que possam substituí-las na composição de substratos e demais produtos.

A fibra de coco aparece como uma alternativa para a produção de substratos, sendo de fácil obtenção e baixo custo, por ser resíduo da exploração comercial da água de coco, existente em abundância. Suas potencialidades de uso na composição de substrato agrícola têm sido abordadas (Carrijo et al., 2002; Meerow, 1997) destacando-se: a capacidade de retenção de água; boa drenagem; acidez; alta salinidade - decorrente, sobretudo, dos altos teores de potássio e cloro; e variação nos teores de nutrientes (Handreck, 1993; Evans et al., 1996; Konduru \& Evans, 1999; Abad et al., 2002). Pesquisas sobre o cultivo de diferentes espécies de plantas ornamentais com uso da fibra, pura ou em mistura, têm mostrado seu desempenho superior a outros substratos (Meerow, 1994; Meerow, 1995; Abad et al., 1997; Mak \& Yeh, 2001). Demattê \& Vitti (1997) avaliando a variação das concentrações de nutrientes ao longo do tempo em diferentes substratos para o cultivo de orquídeas epífitas, recomendaram o uso de blocos de fibra de coco para substituir a fibra de xaxim.

Em estudos com Bromeliaceae, D‘Andréa \& Demattê (2000) e Demattê \& Oshiro (2001) avaliaram substratos alternativos ao xaxim para cultivo de Aechmea fasciata e observaram que a mistura composta de $45 \%$ de casca de coco, $45 \%$ de bagaço de cana e $10 \%$ de húmus pode ser recomendada. Da mesma forma, Demattê $(2001,2002)$ verificou que fibra de coco, casca de pinus e húmus poderiam substituir o xaxim na composição dos substratos para cultivo de Tillandsia gardneri, sendo que as plantas em substratos contendo maior porcentagem de fibra de coco apresentaram maior número de folhas, em relação àquelas cultivadas em xaxim.

Um outro aspecto a ser considerado é a escassez de literatura técnica sobre adubação de bromélias. A adubação foliar é prática comum no cultivo, embora realizada de maneira empírica quanto à formulação, freqüência e dosagem (Andrade \& Demattê, 1999), havendo, portanto, necessidade de estudo a este respeito.

O objetivo do presente trabalho foi comparar o efeito da fibra de coco verde (mesocarpo de coco triturado e mesocarpo de coco triturado lavado) àquele da fibra de xaxim sobre o crescimento e teores de nutrientes de Cryptanthus sinuosus L. B. Smith (Bromeliaceae) sob três níveis de adubação foliar.

\section{MATERIAL E MÉTODOS}

Foram utilizadas mudas de bromélia de $C$. sinuosus L. B. Smith produzidas in vitro no Departamento de Biologia Celular e Genética, do Instituto de Biologia da Universidade do Estado do Rio de Janeiro (UERJ).

$\mathrm{O}$ delineamento experimental foi o de blocos ao acaso, no esquema fatorial (3x3), com quatro repetições, sendo utilizados três substratos (xaxim-X, mesocarpo de coco triturado - MC e mesocarpo de coco triturado lavado MCL) e três níveis de adubação foliar (Níveis 1, 2 e 3). A parcela experimental constou de três vasos, cada um contendo uma planta. A composição da solução de Hoagland ${ }^{\circledR}$ (Hoagland \& Arnon, 1950) foi adotada como base para adubação, variando-se a concentração de NPK. O nível 1 correspondeu à metade $(50 \%)$ da concentração de NPK de Hoagland, o nível 2 correspondeu à concentração de NPK da formulação original $(100 \%)\left(210 \mathrm{mg} \mathrm{L}^{-1}\right.$ de $\mathrm{N}, 31$ $\mathrm{mg} \mathrm{L}^{-1}$ de P e $234 \mathrm{mg} \mathrm{L}^{-1}$ de K) e o nível 3 correspondeu a 1,5 vez a concentração $(150 \%)$ de NPK. A adubação foi realizada a cada 15 dias, durante o período experimental de nove meses, pulverizando-se inicialmente $10 \mathrm{~mL}$ de solução nutritiva nas folhas de cada planta, sendo aumentado o volume conforme o crescimento das plantas até $15 \mathrm{~mL}$. Os volumes foram predeterminados de maneira a molhar completamente as folhas sem haver escorrimento para o substrato, interrompendo-se a pulverização quando as folhas apresentavamse cobertas por uma tênue película de umidade. Não foi usado espalhante adesivo. As condições de temperatura e umidade relativa do ar foram monitoradas durante todo o período experimental.

Para o preparo dos substratos de mesocarpo de coco, o material foi coletado em pontos de venda de água-decoco na cidade de Campos dos Goytacazes na região Norte Fluminense. Após o descascamento do coco, utilizou-se o mesocarpo, que foi triturado em picadeira de forragem (Nogueira/ EM-9F 3A), por três vezes. Após a moagem, o material foi seco ao sol, sobre uma tela do tipo Sombrite estendida sobre suporte de madeira. Uma parte do mesocarpo, após ser triturado, foi colocada em um saco permeável dentro de um tambor, para lavagem com água 
corrente por 24 horas, visando atingir condutividade elétrica próxima à observada para o xaxim e, em seguida, foi seca ao ar como descrito anteriormente. O xaxim utilizado foi adquirido comercialmente. Amostras de cada um dos substratos foram coletadas para avaliação de sua composição mineral.

As mudas foram plantadas em vasos plásticos com capacidade de $0,5 \mathrm{~L}$ contendo os respectivos substratos, sendo uma planta por vaso. Em cada uma das três plantas da parcela foram avaliados: o número de folhas, o diâmetro da roseta e a massa da matéria seca das folhas e raízes. Além disso, foram determinados os teores de nutrientes das folhas e raízes.

Após nove meses de cultivo, os substratos e as folhas das plantas foram submetidos à secagem em estufa com ventilação forçada a $70^{\circ} \mathrm{C}$ por $48 \mathrm{~h}$ e à trituração em moinho Wiley, com peneira de 20 mesh. Em seguida, o material foi submetido às digestões sulfúrica e nitroperclórica, separadamente, segundo metodologias descritas por Malavolta et al. (1997). Os extratos foram utilizados para a determinação dos teores de nutrientes ( $\mathrm{N}, \mathrm{P}, \mathrm{K}, \mathrm{S}, \mathrm{Cl}$ e $\mathrm{Na}$ ). $\mathrm{O}$ pH e a condutividade elétrica (CE) dos substratos foram determinados em água conforme a metodologia proposta por Tedesco et al. (1985).

$\mathrm{O}$ nitrogênio foi determinado usando-se o método de Nessler (Jackson, 1965), o fósforo pela redução do complexo fosfo-molíbdico pela vitamina C (Braga \& Defelipo, 1974); para o nitrato utilizou-se a metodologia descrita por Cataldo et al. (1975). O cloro foi determinado por titulometria (Malavolta et al., 1997). Os teores de potássio e de sódio foram determinados por espectrometria de absorção atômica. O enxofre foi determinado por turbidimetria.

Para fins de análise estatística, os dados observados em cada uma das três plantas da parcela foram usados procedendo-se à análise de variância. As comparações de médias foram efetuadas utilizando-se o teste de Tukey ao nível de $5 \%$ de significância.

\section{RESULTADOS E DISCUSSÃO}

As plantas cultivadas em xaxim $(\mathrm{X})$ apresentaram maior número de folhas

Tabela 1. Diâmetro da roseta, número de folhas, massa da matéria seca (MS) das folhas e raízes, e relação MS folhas/MS raiz de plantas de Cryptanthus sinuosus, cultivadas em xaxim (X), mesocarpo de coco triturado lavado (MCL) e mesocarpo de coco triturado (MC), sob três níveis de adubação foliar (50, 100 e 150\% da concentração NPK da solução de Hoagland), nove meses após o plantio. Rio de Janeiro, UERJ, 2004.

\begin{tabular}{|c|c|c|c|c|c|}
\hline \multirow{2}{*}{ Variáveis } & \multirow{2}{*}{ Substratos } & \multicolumn{3}{|c|}{ Níveis de adubação foliar } & \multirow{2}{*}{ Média } \\
\hline & & 1 & 2 & 3 & \\
\hline \multirow{4}{*}{$\begin{array}{l}\text { Diâmetro } \\
\text { (cm) }\end{array}$} & $x$ & 29,89 & 28,40 & 27,87 & $28,72 a^{*}$ \\
\hline & $\mathrm{MCL}$ & 30,62 & 29,98 & 28,78 & $29,79 \mathrm{a}$ \\
\hline & $\mathrm{MC}$ & 32,09 & 27,14 & 31,21 & $30,15 a$ \\
\hline & Média & 30,87 & 28,51 & 29,29 & \\
\hline \multirow{4}{*}{$\begin{array}{l}\text { Folhas } \\
\text { por } \\
\text { planta }\end{array}$} & $x$ & 21,16 & 20,98 & 22,09 & $21,40 a$ \\
\hline & $\mathrm{MCL}$ & 19,24 & 19,33 & 19,97 & $19,52 \mathrm{~b}$ \\
\hline & $\mathrm{MC}$ & 18,45 & 18,74 & 19,18 & $18,79 b$ \\
\hline & Média & 19,62 & 19,69 & 20,41 & \\
\hline \multirow{4}{*}{$\begin{array}{l}\text { MS } \\
\text { Folhas } \\
\text { (g planta }^{-1} \text { ) }\end{array}$} & $x$ & 7,88 & 7,81 & 8,24 & $7,98 \mathrm{a}$ \\
\hline & $\mathrm{MCL}$ & 6,97 & 6,83 & 7,40 & $7,07 \mathrm{ab}$ \\
\hline & $\mathrm{MC}$ & 6,50 & 6,24 & 6,86 & $6,53 \mathrm{~b}$ \\
\hline & Média & 7,12 & 6,96 & 7,50 & \\
\hline \multirow{4}{*}{$\begin{array}{l}\text { MS } \\
\text { Raízes } \\
{\left.\text { (g planta }{ }^{-1}\right)}\end{array}$} & $x$ & 1,18 & 1,23 & 1,28 & $1,23 a$ \\
\hline & $\mathrm{MCL}$ & 1,00 & 1,09 & 1,16 & $1,08 a b$ \\
\hline & $\mathrm{MC}$ & 1,00 & 0,98 & 0,95 & $0,98 \mathrm{~b}$ \\
\hline & Média & 1,06 & 1,10 & 1,13 & \\
\hline \multirow{4}{*}{$\begin{array}{l}\text { MS folha / } \\
\text { MS raiz }\end{array}$} & $x$ & 7,41 & 7,04 & 8,70 & $7,72 \mathrm{a}$ \\
\hline & $\mathrm{MCL}$ & 7,60 & 7,17 & 8,20 & $7,66 \mathrm{a}$ \\
\hline & $\mathrm{MC}$ & 7,13 & 7,04 & 8,10 & $7,42 \mathrm{a}$ \\
\hline & Média & 7,38 & 7,08 & 8,33 & \\
\hline
\end{tabular}

*Médias seguidas da mesma letra não diferem a 5\% de probabilidade pelo teste de Tukey.

em relação àquelas cultivadas em mesocarpo de coco (MC) e mesocarpo de coco lavado (MCL). O diâmetro das plantas cultivadas em $\mathrm{X}$ não diferiram daqueles das plantas em MC e MCL. Todas as plantas em $\mathrm{X}$ apresentaram coloração verde, enquanto as plantas em MC e MCL apresentaram folhas com coloração avermelhada e roseta aberta com folhas dispostas horizontalmente, sendo mais atrativas do ponto de vista ornamental. Desta forma, o uso do MC como alternativa ao uso do $\mathrm{X}$ para o cultivo de Cryptanthus sinuosus seria vantajoso também pelo aspecto ornamental, além dos aspectos econômico e ambiental.

Em pesquisa com Tillandsia gardneri, Demattê (2002) não observou diferenças significativas entre o número de folhas das plantas cultivadas em substratos à base de fibra de coco, em relação ao que continha xaxim. Como as espécies $T$. gardneri e $C$. sinuosus (epífita e terrestre, respectivamente), são bastante distintas, os resultados observados com ambas podem estar relacio- nados às características intrínsecas das espécies, bem como aos teores de nutrientes dos substratos de $T$. gardeneri aos quais adicionou-se húmus de minhoca e/ou macro e micronutrientes.

Entretanto, quando Coutinho et al. (2001) estudaram o crescimento de mudas de $C$. sinuosus, produzidas in vitro, sob condições de campo e telado, com e sem pré-adubação com solução de Hoagland, obtiveram número de folhas, diâmetro de roseta e massa de matéria seca, das plantas sob telado, semelhantes aos observados neste trabalho, embora os substratos utilizados fossem mais ricos em nutrientes em relação ao $\mathrm{X}, \mathrm{MC}$ e MCL, indicando eficiência dos tratamentos da presente pesquisa em promover o crescimento da espécie, corroborando a adequação da fibra de coco para o cultivo de $C$. sinuosus.

As plantas cultivadas em $\mathrm{X}$ apresentaram valores de massa da matéria seca (MS), das folhas e raízes, mais altos que aqueles observados nas plantas em MC (Tabela 1), mas não diferiram estatisticamente daqueles observados nas plan- 
Tabela 2. Teores de nutrientes na matéria seca das folhas e raízes de Cryptanthus sinuosus, cultivadas em xaxim (X), mesocarpo de coco triturado lavado (MCL) e mesocarpo de coco triturado (MC), sob três níveis de adubação, nove meses após o plantio. Rio de Janeiro, UERJ, 2004.

\begin{tabular}{|c|c|c|c|c|c|c|c|c|c|c|}
\hline \multirow{3}{*}{$\begin{array}{l}\text { Nutrientes } \\
\left(\mathbf{g ~ K g}^{-1}\right)\end{array}$} & \multirow{3}{*}{ Substratos } & \multicolumn{6}{|c|}{ Níveis de adubação } & \multirow{2}{*}{\multicolumn{3}{|c|}{ Média }} \\
\hline & & \multicolumn{2}{|c|}{1} & \multicolumn{2}{|c|}{2} & \multicolumn{2}{|c|}{3} & & & \\
\hline & & Folha & Raiz & Folha & Raiz & Folha & Raiz & Folha & Raiz & \\
\hline \multirow{4}{*}{$N$} & $x$ & 5,29 & 6,18 & 4,63 & 7,04 & 4,60 & 6,99 & $4,84 a^{*}$ & $6,74 \mathrm{a}$ & \\
\hline & $\mathrm{MCL}$ & 3,97 & 5,91 & 3,97 & 5,52 & 4,30 & 6,48 & $4,08 \mathrm{~b}$ & 5,97 a & \\
\hline & MC & 3,96 & 5,01 & 3,86 & 4,70 & 3,97 & 5,27 & $3,93 \mathrm{~b}$ & $4,99 \quad b$ & b \\
\hline & Média & 4,41 & 5,70 & 4,15 & 5,75 & 4,29 & 6,25 & & & \\
\hline \multirow{4}{*}{$\mathrm{P}$} & $x$ & 1,16 & 0,55 & 0,93 & 0,66 & 1,07 & 0,64 & $1,05 \mathrm{a}$ & $0,62 \quad b$ & $\mathrm{~b}$ \\
\hline & $\mathrm{MCL}$ & 0,68 & 0,78 & 0,74 & 0,67 & 0,77 & 0,82 & $0,73 \quad b$ & 0,76 a & \\
\hline & MC & 0,90 & 0,61 & 0,95 & 0,57 & 0,78 & 0,63 & $0,88 \quad b$ & $0,60 \mathrm{~b}$ & b \\
\hline & Média & 0,91 & 0,65 & 0,87 & 0,63 & 0,87 & 0,70 & & & \\
\hline \multirow{4}{*}{ K } & $x$ & 16,12 & 2,59 & 14,82 & 3,37 & 15,43 & 3,30 & $15,46 \mathrm{a}$ & $3,09 \quad b$ & $b$ \\
\hline & $\mathrm{MCL}$ & 11,88 & 2,90 & 12,55 & 2,96 & 12,55 & 3,65 & $12,33 \mathrm{~b}$ & 3,17 & b \\
\hline & MC & 14,47 & 4,81 & 15,80 & 3,97 & 15,14 & 4,26 & $15,14 \mathrm{a}$ & $4,34 \mathrm{a}$ & \\
\hline & Média & 14,16 & 3,43 & 14,39 & 3,43 & 14,37 & 3,74 & & & \\
\hline \multirow{4}{*}{$\mathrm{S}$} & $x$ & 1,10 & 2,00 & 1,14 & 2,15 & 1,02 & 2,09 & $1,09 \mathrm{a}$ & $2,08 \mathrm{a}$ & \\
\hline & MCL & 0,72 & 1,80 & 0,69 & 1,98 & 0,78 & 1,70 & $0,73 \mathrm{~b}$ & $1,83 a$ & \\
\hline & MC & 0,82 & 1,53 & 0,78 & 1,53 & 0,77 & 1,50 & $0,79 \mathrm{~b}$ & 1,52 & $b$ \\
\hline & Média & 0,88 & 1,78 & 0,87 & 1,89 & 0,86 & 1,77 & & & \\
\hline \multirow{4}{*}{$\mathrm{NO}_{3}$} & $x$ & 0,33 & & 0,34 & & 0,33 & & $0,33 \mathrm{a}$ & & \\
\hline & $\mathrm{MCL}$ & 0,29 & & 0,30 & & 0,31 & & $0,30 \quad b$ & & \\
\hline & MC & 0,30 & & 0,28 & & 0,29 & & $0,29 \quad b$ & & \\
\hline & Média & 0,31 & & 0,31 & & 0,31 & & & & \\
\hline \multirow{4}{*}{$\mathrm{Cl}$} & $x$ & 9,32 & & 8,25 & & 8,11 & & $8,56 \mathrm{a}$ & & \\
\hline & $\mathrm{MCL}$ & 6,69 & & 7,62 & & 7,16 & & $7,16 \quad b$ & & \\
\hline & MC & 8,23 & & 8,61 & & 7,72 & & $8,19 a b$ & & \\
\hline & Média & 8,08 & & 8,16 & & 7,66 & & & & \\
\hline \multirow{4}{*}{$\mathrm{Na}$} & $x$ & 2,34 & 0,39 & 2,42 & 0,40 & 2,12 & 0,40 & $2,29 \quad b$ & 0,40 & $b$ \\
\hline & $\mathrm{MCL}$ & 2,53 & 0,48 & 2,85 & 0,50 & 2,62 & 0,41 & $2,67 \quad b$ & 0,46 & b \\
\hline & MC & 3,10 & 0,55 & 3,29 & 0,50 & 3,05 & 0,61 & 3,15 a & $0,55 a$ & \\
\hline & Média & 2,66 & 0,47 & 2,85 & 0,47 & 2,60 & 0,47 & & & \\
\hline
\end{tabular}

*Médias seguidas da mesma letra não diferem a $5 \%$ de probabilidade pelo teste de Tukey.

tas em MCL. Para as mesmas variáveis, as plantas cultivadas em MC e MCL não diferiram entre si, indicando que a alta condutividade elétrica encontrada no substrato MC não prejudicou o acúmulo de matéria seca das plantas. Isto poderia ser explicado pelo fato de a planta ser uma espécie de restinga e estar adaptada a ambientes com elevado teor de sais. Esses resultados, aliados aos dados de número de folhas e diâmetro da roseta, indicam que a lavagem do $\mathrm{MC}$ não seria necessária para o cultivo da espécie e confirmam a viabilidade de seu uso como substituto ao xaxim.

Os resultados das características de crescimento (Tabela 1) e dos teores de nutrientes das plantas (Tabela 2) demonstraram que, independentemente do substrato de cultivo, não houve diferença estatística entre os três níveis de adu- bação foliar (50, 100 e $150 \%$ da concentração de NPK da solução de Hoagland), indicando que pode haver uma economia de nutrientes ao se utilizar metade da concentração de NPK da fórmula original.

Os baixos teores de nutrientes encontrados no substrato MCL não prejudicaram as características de crescimento das plantas (Tabelas 2 e 3 ).

Os teores de $\mathrm{K}$ encontrados nas folhas das plantas cultivadas em X e MC (Tabela 2) não diferiram entre si, embora o teor do nutriente no substrato $\mathrm{X}$ fosse muito mais baixo que no MC (Tabela 3), indicando que os teores foliares observados nas plantas podem ser oriundos da adubação e não do substrato.

Da mesma forma, as plantas cultivadas em MC apresentaram teores de cloro semelhantes àqueles encontrados nas plantas cultivadas em MCL e X. Estes resultados evidenciam que o alto teor de cloro encontrado nas plantas não estava relacionado ao seu cultivo em MC. É possível, entretanto, que o cloro tenha sido proveniente, da água de irrigação utilizada, fornecida pela rede local. Assim, o teor de cloro das plantas em $\mathrm{X}$ poderia estar relacionado à perda de água mais lenta que ele apresenta em relação ao MC (Souza, 2002) e, logo, à maior contribuição da absorção radicular do nutriente para o teor observado nas plantas cultivadas naquele substrato.

As plantas cultivadas em $\mathrm{X}$ apresentaram maiores teores foliares de $\mathrm{N}, \mathrm{P}$ e $\mathrm{S}$ (Tabela 2), justificando-se os maiores valores de número de folhas, massa de 
Tabela 3. Teores de nutrientes, $\mathrm{pH}$ e condutividade elétrica (CE) dos substratos mesocarpo de coco triturado (MC), mesocarpo de coco triturado lavado (MCL) e xaxim (X), utilizados para o cultivo de Cryptanthus sinuosus. Rio de Janeiro, UERF.

\begin{tabular}{|c|c|c|c|c|c|c|c|c|c|}
\hline \multirow{2}{*}{ Substrato } & $\mathrm{N}$ (org.) & $\mathbf{P}$ & $\mathrm{K}$ & s & $\mathrm{Na}$ & NO3 & $\mathrm{Cl}$ & \multirow{2}{*}{$\mathrm{pH}$ em $\mathrm{H}_{2} \mathrm{O}$} & \multirow{2}{*}{$\operatorname{CE}\left(\mathrm{d} S \mathrm{~m}^{-1}\right)$} \\
\hline & & & & $\mathbf{g ~ k g}^{-1}$ & & & & & \\
\hline$x$ & $7,10 a^{*}$ & $0,68 \mathrm{~b}$ & $3,35 \mathrm{~b}$ & $1,31 \mathrm{a}$ & $0,14 \quad c$ & $0,045 \quad b$ & $0,92 \mathrm{~b}$ & $4,00 \mathrm{~b}$ & $1,2 b$ \\
\hline $\mathrm{MCL}$ & $0,67 \mathrm{c}$ & $3,81 \mathrm{a}$ & $1,77 \quad \mathrm{c}$ & $0,62 \mathrm{c}$ & $0,35 \mathrm{~b}$ & 0,12 & $1,89 \mathrm{~b}$ & $4,56 \mathrm{a}$ & $0,7 \mathrm{~b}$ \\
\hline MC & $3,75 \mathrm{~b}$ & $3,81 \mathrm{a}$ & $15,45 \mathrm{a}$ & $0,81 \quad b$ & $2,96 \mathrm{a}$ & $0,42 a$ & 14,25 a & $3,64 \mathrm{c}$ & $6,4 \mathrm{a}$ \\
\hline
\end{tabular}

*Médias seguidas da mesma letra na coluna não diferem a $5 \%$ de probabilidade pelo teste de Tukey.

matéria seca das folhas e raízes (Tabela 1) encontrados nessas plantas. Como o fornecimento de nutrientes foi o mesmo para as plantas cultivadas nos outros substratos, o $\mathrm{X}$ deve ter oferecido alguma outra condição, como disponibilidade de água, que tenha favorecido à absorção adicional dos nutrientes nele contidos, além do fornecido na adubação foliar.

Apesar de as plantas cultivadas em $\mathrm{X}$ terem apresentado teores foliares mais altos para alguns nutrientes (Tabela 2), os teores observados nas plantas de todos os tratamentos testados apresentaram-se abaixo dos níveis considerados suficientes por Jones Junior et al. (1991) para bromeliáceas como Aechmea fasciata e Ananas comosus. Entretanto, as espécies citadas em Jones Junior et $a l .$, (1991) são plantas de porte grande, enquanto $C$. sinuosus apresenta porte reduzido e ocorre em restingas, indicando possuir mecanismos que permitam sua sobrevivência sob condições de baixa disponibilidade e/ou menor absorção de nutrientes para seu crescimento. Além disso, as plantas de $C$. sinuosus, em todos os tratamentos apresentavam grande número de brotações, que podem ter influenciado nos baixos teores observados, uma vez que funcionariam como drenos das folhas.

Altos teores de $\mathrm{K}$, $\mathrm{Na}$ e $\mathrm{Cl}$ foram encontrados no substrato MC (Tabela 3), porém as plantas nele cultivadas não diferiram das cultivadas em $\mathrm{X}$ quanto ao teor foliar de $\mathrm{K}$, nem daquelas em MCL quanto ao teor foliar de $\mathrm{Cl}$ (Tabela 2), indicando que os mesmos estavam relacionados a algum outro fator, além do teor presente no substrato. Abad et al. (2002) discorrem sobre possíveis efeitos prejudiciais da utilização da fibra de coco, em função de seus teores elevados de $\mathrm{Na}$ e $\mathrm{Cl}$, no cultivo de espécies sensíveis à salinidade. Na presente pesquisa, não foram detectados efeitos prejudicais dos teores desses nutrientes sobre o crescimento das plantas, possivelmente por se tratar de uma espécie oriunda de restinga.

Handreck (1993) afirma que a fibra de coco apresenta alta concentração de $\mathrm{K}$, $\mathrm{Na}$ e $\mathrm{Cl}$, mas que o K não possui efeito negativo sobre o crescimento das plantas em geral, podendo ser uma característica positiva do material dispensando sua suplementação. De acordo com o mesmo autor, o $\mathrm{Na}$ é lixiviado facilmente pela água de irrigação. Os teores de $\mathrm{Na}, \mathrm{K}$ e $\mathrm{Cl}$ encontrados no MCL (Tabela 3) no presente trabalho mostram que não só o $\mathrm{Na}$, mas também o $\mathrm{K}, \mathrm{Cl}$ e $\mathrm{O} \mathrm{NO}_{3}^{-}$foram facilmente lixiviados do $\mathrm{MC}$. Os altos teores de $\mathrm{Cl}$ no $\mathrm{MC}$ podem ser explicados pelo fato de $85 \%$ do cloro do fruto de coco verde ser encontrado na casca (Carsalade, 1992).

As plantas cultivadas em $\mathrm{X}$ tiveram raízes com teores de $\mathrm{N}$ e $\mathrm{S}$ semelhantes àqueles de plantas cultivadas em MCL, e superior àqueles das plantas no $\mathrm{MC}$ (Tabela 2). As plantas cultivadas em X e MCL apresentaram raízes com maior massa da matéria seca (Tabela 1) e maiores teores de N (Tabela 2). Embora os teores de $\mathrm{N}$ nas raízes tenham sido próximos àqueles das folhas e, em valores absolutos, maiores, a distribuição relativa do elemento na planta foi a esperada, ou seja, maior nas folhas (em torno de $85 \%$ ) e menor nas raízes (em torno de $15 \%$ ).

Com base nos resultados obtidos pode-se concluir que a fibra de coco, ou mesocarpo verde, pode ser uma alternativa ao uso do xaxim para o cultivo de Cryptanthus sinuosus, promovendo arquitetura da planta (diâmetro e disposição) e coloração mais atrativa das folhas, embora promova menor número de folhas e massa de matéria seca. Os dife- rentes níveis de adubação foliar empregados não causaram efeitos diferenciados nos resultados.

\section{AGRADECIMENTOS}

Ao Setor de Nutrição Mineral LFIT/ CCTA/UENF; LABMIT/UERJ; FENORTE/TECNORTE.

\section{REFERÊNCIAS}

ABAD M; NOGUERA P; NOGUERA V. 1997. Crecimiento de plantas ornamentales de hoja en substratos de cultivo a base de fibra de coco. DEMATTÊ, MESP; VITTI, GC. Variação nas concentrações de nutrientes em substratos vegetais para cultivo de orquídeas epífitas. In: CONGRESSO IBERICO DE CIENCIAS HORTICOLAS, 3. Comunicações... Vilamoura: Associação Portuguesa de Horticultura. p. 76-81.

ABAD M; NOGUERA P; PUCHADES R; MAQUIERIRA A; NOGUERA V. 2002. Physico-chemical and chemical properties of some coconut coir dusts for use as peat substitute for containerized ornamental plants. Bioresource Technology 82: 241-245.

ANDRADE FSA; DEMATTÊ MESP. 1999. Estudo sobre produção e comercialização de bromélias nas regiões sul e sudeste do Brasil. Revista Brasileira de Horticultura Ornamental 5: 97-110.

BENZING DH. 2000. Bromeliaceae: profile of an adaptive radiation. Cambridge: University Press. 690p.

BRAGA JM; DEFELIPO BV. 1974. Determinação espectrofotométrica de fósforo em extratos de solos e material vegetal. Revista Ceres 21: 73-85.

BRASIL. Ministério do Meio Ambiente. Conselho nacional do Meio Ambiente. 2001, 18 de julho. Dispõe contra corte e exploração de espécies ameaçadas de extinção da flora da Mata Atlântica. Disponível em htpp:// www.mma.gov.br/port/conama/res/res01/ res27801.html. Acessado em 11 de março de 2002.

CÂNDIDO MSD. 1995. Chave artificial para o gênero Cryptanthus. Revista da Sociedade Brasileira de Bromélias 2: 15-21.

CARRIJO OA; LIZ RS; MAKISHIMA N. 2002. Fibra de coco verde como substrato agrícola. Horticultura Brasileira 20: 533-535. 
CARSALADE H. 1992. Coconut. Oléagineux 47 327-346.

CATALDO DA; HAROON M; SCHADER LE; YOUNG UL. 1975. Rapid colorimetric determination of nitrate in plant tissue by nitration of salicylic acid. Communication in Soil Science and Plant Analyses 6: 71 - 80.

COUTINHO JC; PORTO RA; DRUMOND L; SILVA JJL; MANSUR E; CARNEIRO LA 2001. Análise do crescimento de mudas produzidas in vitro de Cryptanthus sinuosus no campo e sob telado. In: CONGRESSO NACIONAL DE BOTÂNICA, 52 Resumos...João Pessoa: SBB. p.19-20.

D‘ANDRÉA JC; DEMATTÊ MESP. 2000. Effect of growing media and fertilizers on the early growth of Aechmea fasciata Bak. Acta Horticulturae 511: 271-276.

DEMATTÊ MESP. 2001. Cultivo de Tillandsia gardneri Lndl. em diferentes substratos. In: CONGRESSO BRASILEIRO DE FLORICULTURA E PLANTAS ORNAMENTAIS 13. Resumos... São Paulo:SBFPO. p. 118.

DEMATTÊ MESP. 2002. Cultivo de Tillandsia gardneri Lindl. (Bromeliaceae) em substratos contendo ou não xaxim. In: CONGRESSO BRASILEIRO DE OLERICULTURA, 42 Resumos...Uberlândia: SOB (CD-ROM).

DEMATTÊ MESP; OSHIRO L. 2001. Substratos e fertilizantes no crescimento e na floração de Aechmea fasciata BAK. (Bromeliaceae). In: CONGRESSO BRASILEIRO DE FLORICULTURA E PLANTAS ORNAMENTAIS, 13. Resumos... São Paulo: SBFPO. p. 107.
DEMATTÊ MESP; VITTI GC. 1997. Variação nas concentrações de nutrientes em substratos vegetais para cultivo de orquídeas epífitas. In: CONGRESSO IBERICO DE CIENCIAS HORTICOLAS, 3. Comunicações.. Vilamoura: Associação Portuguesa de Horticultura. p. 63-68.

EVANS MR; KONDURU S; STAMPS RH. 1996. Source variation in physical and chemical properties of coconut coir dust. HortScience 31:965-967.

HANDRECK KA. 1993. Properties of coir dust, and its use in the formulation of soilless potting media. Communications in Soil Science and Plant Analysis 24: 349-363.

HOAGLAND DR; ARNON DI. 1950. The waterculture method for growing plants without soil. Berkeley: Agriculture Experiment Station. $347 \mathrm{p}$.

JACKSON ML. 1965. Soil chemical analysis. Englewood Cliffs: Prentice-Hall Inc. 498p.

JONES JUNIOR JB; WOLF B; MILLS HA. 1991. Plant analysis handbook. A practical sampling, preparation, analysis, and interpretation guide. Athens: Micro-Macro Publishing Inc. $213 \mathrm{p}$.

KONDURU S; EVANS MR. 1999. Coconut husk and processing effects on chemical and physical properties of coconut coir dust. HortScience. 34: 88-90.

LEME EMC; MARIGO LC. 1993. Bromeliads in the Brazilian wilderness. Rio de Janeiro: Marigo Comunicação. Visual Ltda. 183p.
MAK ATY; YEH DM. 2001. Nitrogen nutrition of Spathiphyllum 'Sensation' grown in Sphagnum peat-and coir-based media with two irrigation methods. HortScience 36: 645-649.

MALAVOLTA E; VITTI GC; OLIVEIRA SA. 1997. Avaliação do estado nutricional das plantas: princípios e aplicações. Piracicaba: POTAFOS. 201p.

MEEROW AW. 1994. Growth of two subtropical ornamentals using coir (coconut mesocarp pith) as a peat substitute. HortScience. 29 1484-1486.

MEEROW AW. 1995. Growth of two tropical foliage plants using coir dust as a container medium amendment. HortTechnology 5: 237 239.

MEEROW AW. 1997. Coir dust, a viable alternative to peat moss. Greenhouse Product News 1: 17-21.

MELO TB. 1996. Bromélias no paisagismo. Revista da Sociedade Brasileira de Bromélias 3: 3-7.

PLANTA-RIO. 2001. Informativo 16: 16.

RAUH W. 1990. The bromeliad lexicon. London: Blandford. 430p.

SOUZA NA. 2002. Utilização mesocarpo de coco verde na produção de tutores e substrato para o cultivo de singônio (Syngonium angustatum Schott). Campos dos Goytacazes: UENF. 52p. (Tese mestrado).

TEDESCO MJ; VOLKWEISS SJ; BOHNEN H. 1985. Análises de solo, plantas e outros materiais. Porto Alegre: UFRGS. 186p. (Boletim técnico). 